\title{
OBSERVATORIO
}

\section{CIBERINFRAESTRUCTURA PARA LAS HUMANIDADES DIGITALES: UNA OPORTUNIDAD DE DESARROLLO TECNOLÓGICO PARA LA BIBLIOTECA ACADÉMICA}

\section{Luis Rodríguez-Yunta}

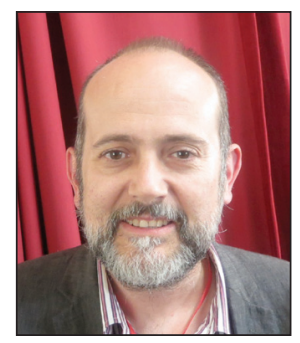

Luis Rodríguez-Yunta, diplomado en formación del profesorado de EGB, licenciado en historia y doctor en documentación por la Universidad Complutense de Madrid (UCM), ha trabajado en los servicios de documentación del Consejo Superior de Investigaciones Científicas (CSIC) desde 1986 -en el ISOC, el Cindoc y actualmente el Centro de Ciencias Humanas y Sociales-. Como representante de estas instituciones ha participado en la red europea Redial y actualmente forma parte de su Junta Directiva. Es profesor asociado en la Facultad de Documentación de la UCM desde septiembre de 2013, y anteriormente realizó esta misma función en el período 2007-2010. Entre 1999 y 2009 fue miembro de la junta directiva de la asociación profesional Sedic, los últimos cuatro años como secretario general.

http://orcid.org/0000-0002-8424-6205

CSIC, Centro de Ciencias Humanas y Sociales, Unidad de Bases de Datos ISOC Albasanz, 26-28. 28037 Madrid, España luis.ryunta@cchs.csic.es

\begin{abstract}
Se destaca el papel central que representan los centros tecnológicos en la consolidación de las humanidades digitales (DH). Para el área de información y documentación, y en especial para las bibliotecas académicas y de investigación, colaborar con las DH supone una oportunidad estratégica de reforzar su ciberinfraestructura. Se defiende que para la consolidación de las DH en España es necesaria la dotación de centros tecnológicos específicos para las humanidades y ciencias sociales.
\end{abstract}

\section{Keywords}

Bibliotecas digitales, Bibliotecas académicas, Bibliotecas universitarias, Humanidades digitales, DH, Ciberinfraestructura, Infraestructura tecnológica, Oportunidades, Desarrollo, Evolución, Tendencias, Futuro, Tecnología, Información y documentación.

Título: Cyberinfrastructure for the digital humanities: an opportunity for technological development in academic libraries

\section{Resumen}

The central role that technology centers play in strengthening the digital humanities (DH) is highlighted. For library and information sciences, and particularly for academic and research libraries, working with DH is a strategic opportunity to enhance their cyberinfrastructure. It is argued that the development of $\mathrm{DH}$ in Spain will require providing technology centers specific to the humanities and social sciences.

\section{Keywords}

Digital libraries, University libraries, Academic libraries, Trends, Future, Digital humanities, DH, Technology, Library and information science, Cyberinfrastructure, Opportunities, Development, Evolution.

Rodríguez-Yunta, Luis (2014). “Ciberinfraestructura para las humanidades digitales: una oportunidad de desarrollo tecnológico para la biblioteca académica". El profesional de la información, septiembre-octubre, v. 23, n. 5, pp. 453-462.

http://dx.doi.org/10.3145/epi.2014.sep.01

\section{Expansión e institucionalización de las humanidades digitales a escala internacional}

Las humanidades digitales (en adelante $\mathrm{DH}$ ) constituyen un campo diverso y aún emergente que no es fácil de definir. Se trata en realidad de un término estratégico (Kirschenbaum,
2012), un marco amplio que abarca tanto el uso de las tecnologías de la información en la investigación en humanidades y ciencias sociales, como la reflexión teórica en torno a las relaciones entre estas disciplinas y las nuevas tecnologías, el cambio cultural y social por la fuerte influencia de internet o las manifestaciones culturales en formato digital. 
Su desarrollo se relaciona con la aplicación de la e-ciencia en el campo de las ciencias sociales y humanas, pero éstas a su vez han aportado características propias (Estalella; Ardévol, 2011): la reflexión epistemológica sobre la aplicación de herramientas tecnológicas en la investigación, la consideración de internet también como un campo de estudio o la reflexión sobre la dimensión ética en el flujo de informaciones y datos a través de los nuevos media.

En la implementación de las $\mathrm{DH}$ no ha dejado de haber un fuerte debate sobre su construcción teórica y sus implicaciones (Kirschenbaum, 2014), y la internacionalización de iniciativas ha contribuido a una mayor imprecisión en su definición (Spence, 2014a). Se configuran como un ámbito transdisciplinar o de colaboración interdisciplinar que integra diferentes líneas y realizaciones tecnológicas aplicadas a cualquier disciplina de humanidades y ciencias sociales. Igualmente pueden considerarse un movimiento internacional, pues cuentan con manifiestos generados en el marco de los encuentros que apoyan su configuración como una comunidad internacional de práctica, multilingüe, multidisciplinar, solidaria, abierta y de libre acceso a datos y metadatos.

\section{3 fue clave para la visibilidad de las humanidades digitales en España}

Pese a esta imagen algo imprecisa, las $\mathrm{DH}$ se han consolidado como un área de actividad científica y académica, con una magnitud internacional. Esta consideración se basa en la presencia de varias características, constatables a nivel global:

- Existencia de asociaciones y congresos de participación abierta. Entre los encuentros internacionales de $\mathrm{DH}$ cabe destacar los conocidos como THATCamp, y entre las asociaciones, la Alliance of Digital Humanities Organizations $(A D H O)$, que integra a otras organizaciones regionales y convoca encuentros anuales. Dentro de esta agrupación se encuentra la European Association for Digital Humanities (EADH), denominación actual de la Association for Literary and Linguistic Computing (ALLC) creada en 1973, y que a su vez cuenta con entidades nacionales en Italia y Alemania. La estructura global de la $A D H O$ convive con agrupaciones de ámbitos disciplinares concretos, por ejemplo Digital Classicist para la comunidad de estudios clásicos.

- Publicación de revistas científicas especializadas de suficiente prestigio y difusión. Pueden destacarse dos que incluyen el concepto de DH en su título, Digital humanities quarterly y Journal of digital humanities, pero la lista puede ampliarse a varias decenas de publicaciones si se tienen en cuenta entornos específicos como lingüística (Literary and linguistic computing), historia (Digital medievalist. The journal of the digital medievalist community) o geografía (Geolnformatica), así como las publicaciones sobre edición digital, bibliotecas digitales o patrimonio digital.

- Presencia en plataformas de comunicación social en internet. Existen blogs específicos (Digital humanities now;
Digital scholarship in the humanities; Filología digital), grupos en Zotero (Digital humanities education) o plataformas wiki sobre recursos (Digital humanities wiki; Dirt - Directory of digital research tools), que muestran la vitalidad de las DH dentro de la web social.

- Oferta de cursos de formación superior. Se organizan masters específicos en universidades de prestigio, como los realizados por King's College London y University College London. Otra modalidad de formación es la de cursos breves, representada por la escuela de verano Digital Humanities Summer Institute, de la University of Victoria en Canadá.

- Institucionalización en centros de referencia, unidades de investigación o centros tecnológicos específicos. La red CenterNet recoge casi 200 instituciones en todo el mundo y promueve un portal para la colaboración (DHCommons). Estos organismos tienen habitualmente su sede en universidades como institutos de investigación, centros interfacultativos o consorcios interuniversitarios. La relación debe completarse con bastantes más instituciones no asociadas a CenterNet. En Estados Unidos existe además una oficina federal desde 2008, la Office of Digital Humanities, dependiente del National Endowment for the Humanities (NEH) que ofrece programas de financiación de proyectos.

\section{Las humanidades digitales en España}

En España las DH cuentan con una dilatada tradición si se tienen en cuenta las iniciativas de aplicación de la tecnología digital a la edición de textos y la investigación, especialmente en el ámbito de la filología (Rojas, 2013). Las Actas del Seminario internacional sobre bibliotecas digitales y bases de datos especializadas para la investigación en literaturas hispánicas (Casas-Del-Álamo et al., 2011) son un buen ejemplo de presentación de nuevos recursos digitales en este campo:
- Bieses - Bibliografía de escritoras españolas;
- Biblioteca Saavedra Fajardo de pensamiento político his- pánico;
- Clarisel - Bases de datos de: Literatura de caballerías (Amadís), Cuento medieval (Sendebar) y Literatura arago- nesa (Heredia);
- Dialogyca, BDDH-Biblioteca digital de diálogo hispánico;
- Bidiso - Biblioteca digital Siglo de oro;
- Nicanto - Obras impresas de escritores del siglo XVIII; o
- Phebo - Poesía hispánica en el bajo barroco.

La asociación Humanidades Digitales Hispánicas comenzó a gestarse en 2011 y celebró su primer congreso en julio de 2013. Hasta ese momento, la dispersión de grupos de investigación interesados en $\mathrm{DH}$ no se había plasmado en la constitución de redes y estructuras de carácter nacional. Aunque en la asociación predominan los investigadores del área filológica, puede representar el principal punto de referencia para la visibilidad de las DH en España.

Este proceso es paralelo al de otros países de la comunidad Iberoamericana, en donde también se han constituido asociaciones recientemente: Red de Humanidades Digitales de México (2011, constituida como asociación en 2014) y Asociación Argentina de Humanidades Digitales (2013). Se- 
ría deseable la colaboración entre estas redes, así como su integración en la estructura internacional de $A D H O$.

El pasado año 2013 ha sido clave para la visibilidad de las DH en España. Además del congreso de la asociación Humanidades Digitales Hispánicas, tuvieron lugar otros dos eventos científicos destacados. Por una parte, el organizado por el grupo de investigación Griso en Pamplona: Humanidades digitales: visibilidad y difusión de la investigación. Por otra, las I Jornadas sobre ciencias sociales y humanidades digitales en la Universidad de Granada. Estos encuentros

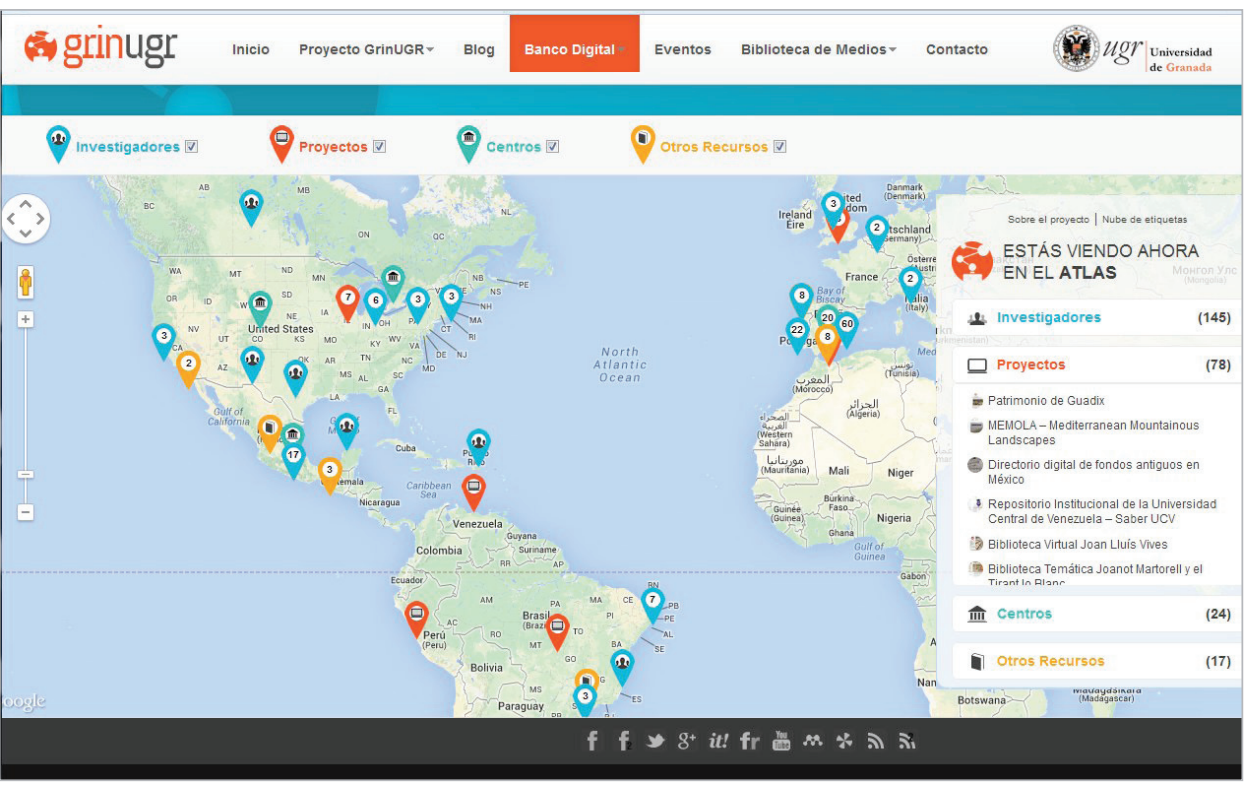

Atlas de ciencias sociales y humanidades digitales http://grinugr.org/mapa han mostrado la confluencia

entre investigadores de humanidades con el ámbito de la comunicación y la edición digital.

En la Universidad de Granada se localiza GrinUGR, grupo de aprendizaje e investigación interdisciplinar que tiene por objetivo el intercambio de experiencias e ideas vinculadas al empleo de internet. Su acrónimo proviene de su primera denominación GRupo de INternet de la Universidad de GRanada, pero actualmente se describe como Co-laboratorio sobre culturas digitales en ciencias sociales y humanidades. Entre sus proyectos ha desarrollado un Atlas de ciencias sociales y humanidades digitales, en el que se reflejan investigadores, centros, proyectos y recursos a escala internacional. Entre las iniciativas españolas que se localizan en este atlas, cabe destacar la Red Aracne - Red de Humanidades Digitales y Letras Hispánicas. Se trata de una red internacional constituida a partir de un seminario organizado en la Universidad Complutense de Madrid por el equipo de la antes citada Dialogyca BDDH, Biblioteca digital de diálogo hispánico. Igualmente pueden citarse ejemplos en disciplinas concretas como la Sociedad Española de Arqueología Virtual o el grupo de investigación Art (Arquitectura i Societat Digital) de la Universitat de Barcelona.

Entre las revistas científicas españolas la publicación que más se acerca a la mención de las DH en su título, es Digithum, Les humanitats en l'era digital, de la Universitat Oberta de Catalunya (UOC). También cabe destacar otras que las citan en su definición de ámbito: Artnodes (UOC), Caracteres. Estudios culturales y críticos de la esfera digital (editada en Salamanca) o Janus. Estudios sobre el Siglo de oro (Universidade da Coruña). Igualmente pueden encontrarse blogs específicos de interés como Filindig de Elena González-Blanco.

En cuanto a la formación superior, la oferta es aún escasa. El Máster en humanidades digitales, de la Universidad de Castilla-La Mancha, celebró su cuarta edición en el curso 2010-2011. Un nuevo máster con la misma denominación se ha iniciado en la Universitat Autònoma de Barcelona en el curso 2013-2014. Por otra parte, la Universidad Nacional de Educación a Distancia (UNED) ha incluido un curso de iniciación a las $\mathrm{DH}$ en su oferta de verano y un título de experto profesional en DH que se inicia en el curso 2014-2015.

Finalmente, el aspecto más débil de la consolidación de las DH en España es la escasa existencia de centros específicos de apoyo tecnológico. El único centro español asociado a CenterNet es el Grupo de Gestión del Conocimiento y Nuevas Tecnologías de la Universidad de Castilla-La Mancha, cuya web no muestra actividad relevante en la actualidad. Pueden citarse algunos centros específicos del campo de la creación artística, por ejemplo MediaLab USAL, que se define como un espacio de encuentro físico y virtual, creado por el Servicio de Innovación y Producción Digital de la Universidad de Salamanca. La mejor noticia en cuanto a la dotación de centros es la creación en 2014 del Laboratorio de Innovación en Humanidades Digitales (LiNHD) en la UNED, un buen modelo de unidad capaz de llevar a cabo proyectos innovadores de investigación.

En contraste con el panorama internacional, no puede decirse que las DH tengan aún suficiente implantación en España. Sí existe una comunidad investigadora interesada, pero faltan más cursos de formación, revistas científicas y, en especial, es necesaria la dotación de centros tecnológicos que trabajen específicamente en ciencias sociales y humanas a fin de apoyar la realización de recursos.

\section{Ciberinfraestructuras para las humanidades y ciencias sociales}

Hace ya 8 años una comisión creada por el American Council of Learned Societies (2006) elaboró un informe sobre la necesidad de desarrollar ciberinfraestructuras para las humanidades y ciencias sociales. El concepto de ciberinfraestructuras había sido utilizado anteriormente en 2003 en un informe elaborado por Dan Atkins para la National Science 
"ili Qué es LINHD Investigación Formación Servicios Herramientas Noticias y eventos Participa Q

PROYECTOS DE INVESTIGACIÓN

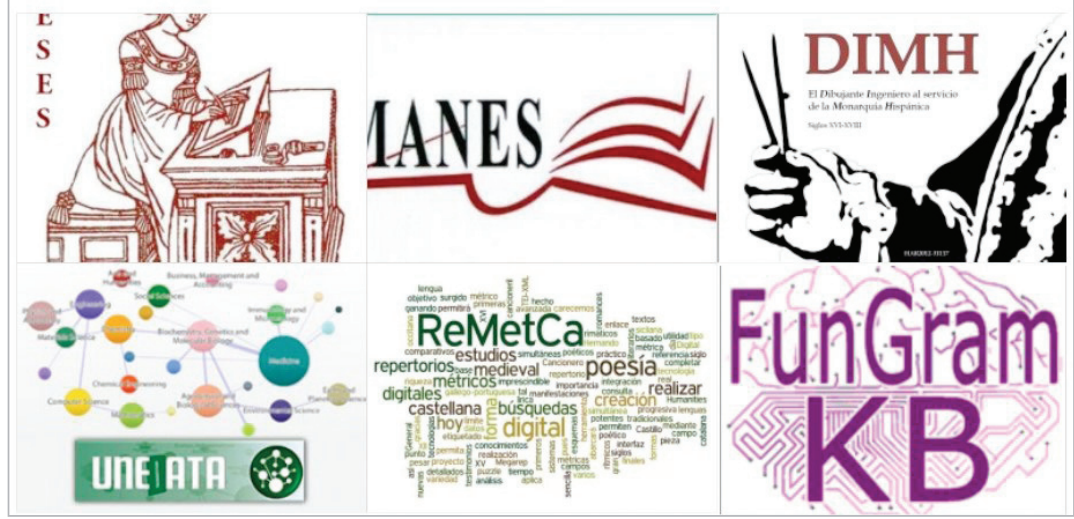

Proyectos de investigación del Laboratorio de Innovación en Humanidades Digitales de la UNED http://linhd.uned.es/investigacion

Foundation, en referencia a los centros de computación, bibliotecas digitales, repositorios de datos, instrumentos y herramientas de software.

Las recomendaciones del ACLS son plenamente aplicables hoy en España. Entre ellas, destaca la defensa de la inversión en ciberinfraestructuras para las humanidades y ciencias sociales como prioridad estratégica, mediante la dotación de centros de apoyo técnico. La creación de estas unidades con entidad propia es el eje central de la consolidación de las DH (Zorich, 2008; Rodríguez-Yunta, 2013). Deben contar con personal académico y técnico no perteneciente a otros departamentos, al margen de que establezcan cooperaciones con grupos y centros de investigación en la realización de proyectos. Las funciones que pueden abordarse desde estas unidades o "centros de humanidades digitales" abarcan diferentes ámbitos:

- Formación dirigida a estudiantes e investigadores: manejo de herramientas, guías de buenas prácticas, orientación sobre proyectos. Garantizan la experiencia para evitar errores cometidos en proyectos anteriores o la selección del software más adecuado.

- Apoyo técnico para proyectos de DH, como alojamiento y creación de portales, repositorio de colecciones digitales, cumplimiento de estándares y preservación a largo plazo. Aportan el desarrollo técnico de proyectos para los grupos de investigación: definición de requisitos técnicos, alojamiento, seguridad y gestión.

- Mantenimiento de recursos generales para proyectos: plataformas de noticias y blogs, sistemas de navegación y búsqueda, modelos de edición digital.

- Realización de aplicaciones a medida para usos específicos dentro de un proyecto de investigación, soluciones tecnológicas adaptadas a las necesidades de las $\mathrm{DH}$.

Puede decirse que estos centros han evolucionado a partir de su precedente, las unidades de informática para las humanidades (humanities computing), ampliando sus objetivos para representar actualmente un papel central en todo el proceso de diseño, desarrollo y mantenimiento a largo o guías para historiadores.

plazo de proyectos de bibliotecas digitales, bases de datos o cultura digital.

Para localizar ejemplos de centros de $\mathrm{DH}$, el principal referente es la red CenterNet, miembro a su vez de $A D H O$. En julio de 2014 figuran en su relación de instituciones 198 centros de 29 países, con un claro predominio de Estados Unidos (78), seguido de Canadá (20), Reino Unido (20), Alemania (12), Australia (9), Francia (8) e Italia (8). Las características de estos organismos son muy diversas, aunque en su mayoría son centros multidisciplinares. Otras unidades desempeñan su función en un área disciplinar concreta. Así por ejemplo, el Roy Rosenzweig Center for History and New Media, en la George Mason University, es un centro de referencia para la historia digital, ha liderado numerosos proyectos de $\mathrm{DH}$, elaboración de software

Algunas de estas unidades son de muy reciente creación. Por ejemplo, el Laboratoire de cultures et humanités digitales (LaDhul) de la Université de Lausanne, organizador del congreso de la $A D H O$ de 2014 , inició sus funciones tan sólo en 2013. Puede mostrarse como modelo de integración entre disciplinas, ya que se trata de un centro interfacultativo que depende de las facultades de ciencias sociales y políticas, letras y teología y ciencias de las religiones.

\section{El aspecto más débil de la consolidación de las humanidades digitales en España es la escasa existencia de centros espe- cíficos de apoyo tecnológico}

Igualmente pueden encontrarse centros muy relevantes que no están citados en CenterNet. Un ejemplo es Huma-Num, institución francesa creada a partir de la fusión de Adonis (creado en 2007, fue el primer tgir dedicado a las humanidades en Francia) y Corpus IR en 2013. Se constituye como una tgir, es decir una "très grande infrastructure de recherche". Esta categoría es ya de por sí un dato destacable, pues muestra como en Francia el concepto de grandes instalaciones científico-técnicas ya abarca las ciencias sociales y humanas, gracias al auge de las $\mathrm{DH}$. Orgánicamente supone dotar a estas disciplinas de una infraestructura básica similar a la función que realizan en el desarrollo científico y tecnológico un gran telescopio, un buque oceanográfico o un gran acelerador de partículas. Entre sus logros se encuentra la plataforma Isidore, disponible desde 2011, y que aporta un enfoque innovador de un motor de búsqueda: permite el enriquecimiento entre metadatos y la información extraída del texto completo. Huma-Num es una estructura organizativa, pero también un espacio de intercambio, de esfuerzo colaborativo. Una de sus líneas de trabajo consiste en la elaboración de guías de buenas prácticas sobre el uso de nuevas tecnologías en humanidades y ciencias sociales: 
edición digital, interoperabilidad de datos, preservación y migración de formatos.

Otra opción es la constitución de consorcios interuniversitarios. Un ejemplo es el Texas Digital Humanities Consortium, que da servicio a seis universidades. La formación de este tipo de unidades entre varias instituciones permite potenciar la colaboración y organizar encuentros regularmente.

\section{La ciberinfraestructura, más allá del consumo de tecnología}

El desarrollo de las DH puede interpretarse como la transferencia a las humanidades del paradigma de la eciencia y en consecuencia de la importancia de las ciberin-

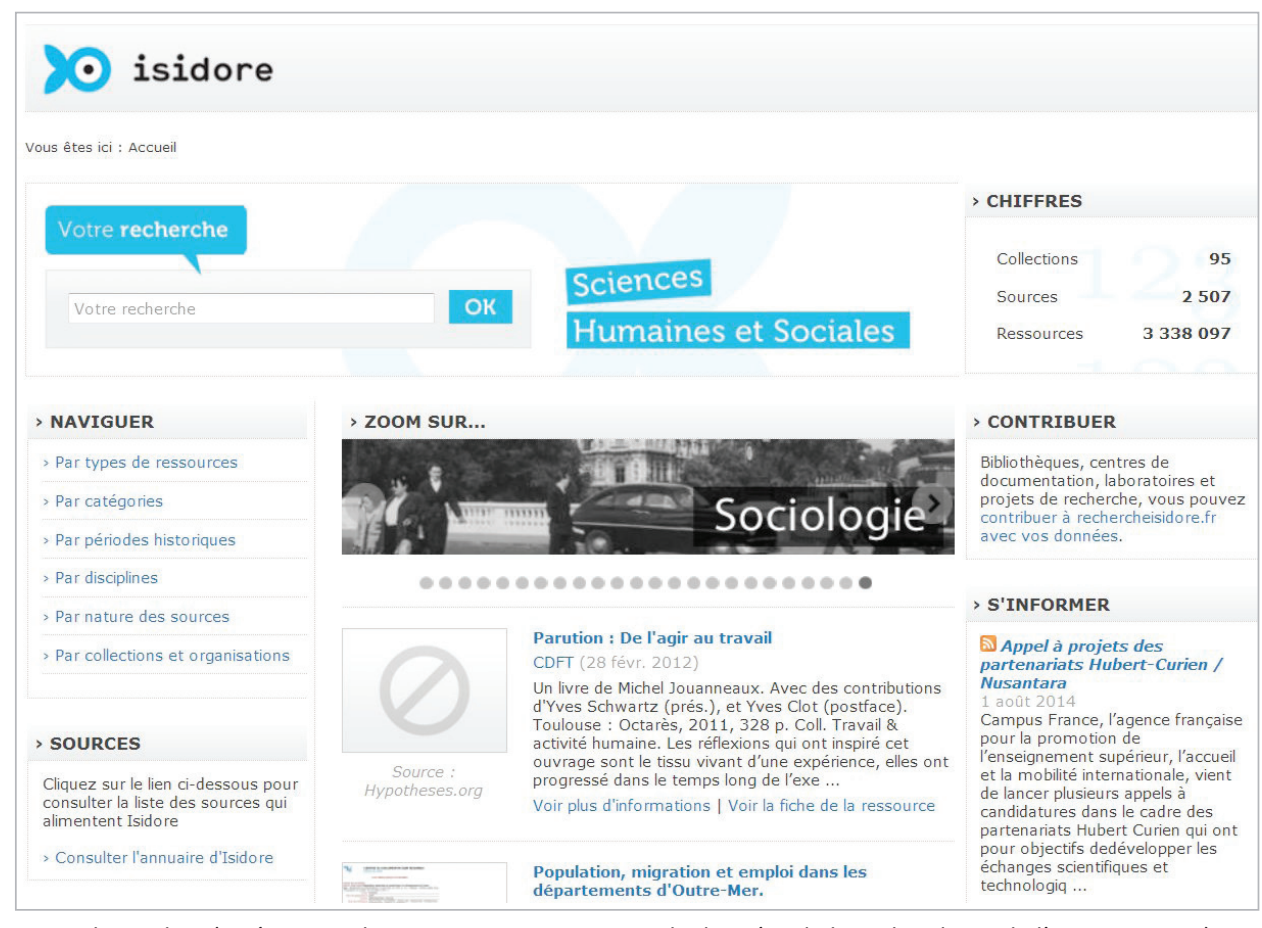

Buscador Isidore (Intégration de services, interconnexion de données de la recherche et de l'enseignement) http://www.rechercheisidore.fr fraestructuras tecnológicas para su implementación (Anderson; Blanke, 2012; Romero, 2014). La dotación de centros tecnológicos es sin duda el factor clave en este proceso.

Por supuesto las infraestructuras tecnológicas ya existen en el entorno de trabajo de los humanistas y científicos sociales en cualquier universidad o centro de investigación, pero ¿son suficientes para garantizar siquiera la adaptabilidad de las instituciones a las necesidades futuras? ¿Son capaces de sostener proyectos de innovación? ¿Son infraestructuras diseñadas exclusivamente para sobrevivir o permiten un crecimiento sostenible?

\section{La infraestructura tecnológica debe es- tar en un contexto que incluya filosofía de cambio y humanismo}

La relación entre tecnología e investigación es ya un hecho irrenunciable. Cualquier actividad científica va a utilizar herramientas tecnológicas. Pero la etiqueta de "humanista digital" no debe aplicarse a un mero usuario de tecnología, debe aportar también capacidad de innovación (VanZundert, 2012). Se trata de un agente de creación de conocimiento, que puede hablar con autoridad en el terreno tecnológico y ejercer un cierto liderazgo en las relaciones entre sociedad y tecnología. Un objetivo tan ambicioso precisa apoyo institucional, formar parte de una gran entidad o un consorcio sólido.

Infraestructura tecnológica, sí, pero dentro de un contexto que incluye otros dos elementos: filosofía de cambio y énfasis en el factor humano. En resumen tecnología + innovación + humanismo. Efectivamente las $\mathrm{DH}$ no son sólo tecnología, la ciberinfraestructura es el punto de partida, no la finalidad. El énfasis en la parte tecnológica no debe hacer olvidar que las $\mathrm{DH}$ se caracterizan también por el enfoque humanístico: reflexión sobre las interacciones humanas y la capacidad de transformación social.

Las DH suponen también una respuesta a la aparición de nuevos públicos (Spence, 2014b). El cambio cultural implica una nueva relación entre creador y público, tanto para el escritor o el artista, como para el investigador. El conocimiento se hace colaborativo. Este discurso puede hacerse realidad en el medio académico si se cuenta con la estructura tecnológica necesaria y se lleva a cabo un cambio generacional en el profesorado.

\section{Las $\mathrm{DH}$, una oportunidad para dotar de infraestructuras para la investigación en información y documentación y para las bibliotecas académicas}

Ante todo hay que ver a las $\mathrm{DH}$ como una gran oportunidad en la que actuar colectivamente. Así se señala para campos como el hispanismo (Lucía-Megías, 2014). La oportunidad es también de especial interés para los departamentos de biblioteconomía y documentación, de cara a:

- Reivindicar un mayor protagonismo de la información y documentación (library and information science) entre las ciencias sociales y humanas, en la difusión social y también en la innovación e investigación.

- Expandir los campos de estudio y métodos de investigación adaptados al nuevo entorno, métodos de búsqueda en archivos y bibliotecas digitales, tratamiento de datos, visualización...

- Fomentar la interdisciplinariedad, la colaboración en equipos de trabajo en el que participen bibliotecarios o documentalistas, con tareas propias de sus conocimientos, curación de contenidos, preservación... 
Las $\mathrm{DH}$ reflejan la necesidad de las disciplinas humanísticas de reivindicarse en la sociedad de la información. También de reinventarse y asumir la revolución tecnológica en todas sus consecuencias. A las bibliotecas de investigación este proceso no les es ajeno. Desde los primeros pasos en la automatización de sus herramientas de gestión, la tecnología se ha convertido ya en el eje central de toda su actividad. La gestión de servicios online se considera ya tan relevante como la existencia de infraestructuras físicas.

De la extensa nómina de centros de $\mathrm{DH}$, sólo algunos de ellos se localizan en bibliotecas universitarias, por ejemplo el Alabama Digital Humanities Center, el Digital Humanities Center en la Columbia University o el Center for Digital Scholarship en la Brown University. En consecuencia, es natural que muchos de los proyectos de $\mathrm{DH}$ se realicen sin intervención de centros bibliotecarios, incluso cuando consisten en bibliotecas digitales. En su análisis sobre la aportación de las bibliotecas digitales a las DH, Jeffrey A. Rydberg-Cox (2006) selecciona cuatro estudios de caso:

- The valley of the shadow: Two communities in the American civil war,

- Museum of the History of Science in Florence,

- Voyager Mozart cd-rom, y

- Perseus digital library.

Ninguno de estos desarrollos fue liderado o alojado por una biblioteca tradicional.

La biblioteca debe ser un socio indispensable en la aplicación de las tecnologías a la investigación, la enseñanza y la difusión social en ciencias sociales y humanas

Las bibliotecas académicas y de investigación deben buscar un mayor protagonismo en los proyectos de $\mathrm{DH}$, ya que este tipo de productos son un medio natural de colaboración y aprovechamiento de sus propios recursos. El bibliotecario y el humanista digital comparten objetivos, también necesidades. Como proponen Vandegrift y Varner (2013) el profesional de estos centros debe evolucionar para integrarse de forma efectiva en la comunidad académica de las DH. La biblioteca debe ser un "partner" indispensable en los proyectos de aplicación de las tecnologías a la investigación, la enseñanza y la difusión social en ciencias sociales y humanas.

Esta colaboración no debe ser superficial sino engarzarse en profundidad. Sería superficial si la biblioteca se limita a ser depositaria de los resultados de los proyectos, debe aspirar a colaborar en todas las partes del proceso, desde el diseño y la puesta en marcha, hasta la comunicación de resultados.

\section{¿Qué aportan las bibliotecas a los proyectos de DH?}

- Un punto de referencia para centralizar la información sobre proyectos en curso junto a otros ya finalizados. Los investigadores y grupos de investigación tienden a trabajar de forma independiente en función de la obtención de recursos de financiación. Se crean nuevos sitios web que a menudo no tienen garantizada la supervivencia o la sostenibilidad de su producto. Tanto para la localización de proyectos como para albergar y centralizar su mantenimiento en la web más allá de la duración del proyecto, la ubicación adecuada es la biblioteca, o mejor aún el consorcio, el archivo común mantenido por varias instituciones.

- Un punto de acceso para la difusión como recurso de información, para la investigación y la enseñanza. La biblioteca ya cumple esta función, pero debe integrar el acceso a los recursos de su institución que se desarrollan fuera de su ámbito.

- Experiencia conseguida en los últimos años en la difusión del conocimiento, buscando una potenciación del impacto social.

- Amplio conocimiento en la aplicación de estándares para la preservación, el acceso abierto y la interoperabilidad.

- Experiencia en la gestión de derechos de propiedad intelectual.

\section{¿Qué beneficios pueden extraer las bibliotecas de la colaboración en proyectos de $\mathrm{DH}$ ?}

- Abordar la necesidad común de plantear nuevos retos emergentes en preservación y accesibilidad de los datos.

- Desarrollar nuevos modelos en el acceso a la información, nuevas formas de visualización gráfica o sistemas de navegación basados en web semántica. A pesar de la experiencia en organización del conocimiento, las actuales interfaces bibliotecarias dejan aún mucho que desear en cuanto al aprovechamiento eficaz de disponer de una información tan estructurada.

- Garantizar un apoyo tecnológico para cumplir los compromisos adquiridos en cuanto a preservación digital a largo plazo, protección de datos, mantenimiento de servicios para un número elevado de usuarios, usabilidad y accesibilidad de fuentes masivas de información y renovación de formatos para nuevos dispositivos, acordes con la evolución de las demandas sociales y la evolución de las tecnologías.

Hay una motivación extra para promover la participación activa de las bibliotecas académicas y de investigación en los proyectos de $\mathrm{DH}$ : evitar la competencia innecesaria y la duplicidad de esfuerzos. Para optimizar la gestión, los recursos de información deben ubicarse en el entorno de la biblioteca, no al margen de la misma.

\section{Un camino por recorrer: un modelo avanzado en las bibliotecas digitales}

Las bibliotecas físicas han superado en gran parte sus muros tradicionales. De la gestión de la colección se evolucionó a la gestión de servicios y recursos de información. Pero el cambio tecnológico aún está en proceso y la transformación apenas ha comenzado. Por ejemplo, hay una alta expectativa sobre las interfaces en la web semántica y la explotación de los datos abiertos vinculados, que habrá de tener una fuerte repercusión sobre las webs bibliotecarias.

El concepto de biblioteca híbrida (hybrid library o blended library) es un modelo que integra la tradicional colección física con la gestión de recursos digitales. En este contexto, el bibliotecario debe ser un profesional que domine tanto las 
herramientas tradicionales como las nuevas tecnologías. En otros aspectos, el perfil del bibliotecario también tiende a acercarse a la industria editorial, en cuanto que se convierte en un agente activo en el ecosistema de la comunicación académica (Maron et al., 2013).

Las bibliotecas digitales tienen aún un largo camino por recorrer para ser herramientas capaces de generar conocimiento (Hobohm, 2012). De los repositorios como meros contenedores de documentos, debe evolucionarse hacia sistemas de consulta que permitan la interpretación de informaciones y datos, la elaboración de información estructurada extraída de los propios documentos, la minería de datos y el análisis de textos o la generación de nuevas formas de visualización relacionando documentos diversos.

Desde la práctica tradicional, se debe hacer un esfuerzo especial por aplicar una indización en profundidad de los documentos digitalizados (Rodríguez-Yunta, 2014). Las obras colectivas, actas de congresos y compilaciones deben identificarse en todas sus partes. Además, es necesario identificar con metadatos propios todos los elementos de artículos o libros con características independientes de autoría o procedencia, o que puedan estar sujetos a una reutilización al margen del resto del documento: fotografías, ilustraciones, gráficos, tablas, apéndices documentales o ficheros anexos de datos.

Por otra parte, las bibliotecas digitales deben abrirse a la utilización de sistemas de marcado de textos para facilitar su procesamiento automatizado, una de las principales líneas de trabajo en DH (Schmidt, 2012). El estándar TEI (text encoding initiative) se lleva aplicando desde hace ya dos décadas en textos literarios y lingüísticos (Bia-Platas; Sánchez-Quero, 2001). A pesar de haberse establecido pautas en este sentido, la mayor parte de las bibliotecas digitales han optado por la cantidad por encima de la calidad, acumulando documentos en formato pdf sin estructurar.

También en el área de la historia se han implementado herramientas para mejorar la operatividad de bibliotecas digitales. Un ejemplo es el proyecto Omeka del Roy Rosenzweig Center for History and New Media. Se trata de una plataforma open source para la edición de textos enriquecidos y acordes con los estándares, un software a caballo entre la gestión de contenidos web, la gestión de colecciones y los sistemas de gestión de archivos digitales.

Un terreno de gran interés para el desarrollo de las $\mathrm{DH}$ es la integración de datos de patrimonio cultural (Oldman et al., 2014). Los proyectos de web semántica basados en el modelo Cidoc CRM ${ }^{1}$ implican a museos, archivos y bibliotecas. La tecnología de datos vinculados supone una gran oportunidad para que las instituciones del patrimonio cultural desempeñen un papel de liderazgo. La investigación en la representación del conocimiento es un ámbito en el que la museología, la archivística, la biblioteconomía y la documentación mantienen una fuerte tradición, y que también atrae la atención de los centros de DH. La Alliance of Digital Humanities Organizations $(A D H O)$ cuenta con un grupo específicamente dedicado a linked open data, para promocionar el conocimiento de esta línea de trabajo. Igualmente se halla un grupo específico sobre esta temática en el Texas
Digital Humanities Consortium. En España, a las iniciativas de instituciones como la Biblioteca Nacional de España o el Museo del Prado, se han de sumar también las que proceden de centros de DH como el proyecto Unedata del Laboratorio de Innovación en Humanidades Digitales (LiNHD) de la UNED.

Por último, cabe destacar que el libro electrónico constituye un nuevo soporte capaz de generar una revolución cultural similar a la imprenta en el Renacimiento, como una segunda textualidad (Lucía-Megías, 2014). Pero este tópico sólo se hará realidad si se explota en todas sus consecuencias el nuevo medio. La explosión de internet y del acceso abierto está ampliando estas capacidades al facilitar que la intertextualidad, característica del texto digital, se establezca fuera del propio documento, mediante datos abiertos vinculados. Las iniciativas por constituir plataformas editoriales de acceso abierto para las humanidades (OpenEdition, Open Humanities Press) suponen también una oportunidad para abordar de forma colectiva nuevas formas de edición digital.

Los recursos de información deben ubicarse en el entorno de la biblioteca para evitar la competencia innecesaria, la duplicidad de esfuerzos y optimizar la gestión

\section{Conclusiones}

En cualquier entorno, la infraestructura por sí misma no es una garantía suficiente de desarrollo, pero sin ésta cualquier progreso puede resultar meramente aparente y derrumbarse en poco tiempo. Es pues una necesidad, el punto de partida. Las $\mathrm{DH}$ reúnen estas condiciones: son una apuesta segura ya consolidada a nivel internacional y agrupan a una comunidad de investigadores que ya ha demostrado capacidad e iniciativa. Es pues el momento de abordar su institucionalización en países como España, en colaboración con instituciones iberoamericanas y europeas.

Innovación y tecnología son conceptos necesariamente relacionados en la sociedad actual. Pero no podemos considerar innovación a la mera implementación de productos enlatados de software comercial. La capacidad de innovación precisa de una dotación especial de recursos humanos e institucionales. La creación de centros de apoyo tecnológico a las DH supondría una gran oportunidad para el ámbito académico de información y documentación y para el profesional de las bibliotecas, archivos, museos y centros de documentación. En ambos casos es indispensable reforzar su capacidad de innovación tecnológica y asegurar un crecimiento sostenible de sus medios tecnológicos.

Tanto los departamentos de biblioteconomía y documentación como las bibliotecas académicas deben mostrar una actitud más proactiva en el campo de las DH para poder reclamar un rol protagonista: desarrollar sus propios proyectos de ámbito específico y difundirlos en los congresos de $\mathrm{DH}$, crear portales y blogs que describan los proyectos de interés en su institución, mantener directorios de herramientas y recursos, disponer de repositorios de salvaguarda no sólo de documentos y publicaciones sino también de otros 
resultados de la actividad científica (portales, blogs, bases de datos, materiales educativos), incluir entre sus intereses la creación cultural en formato digital...

Dentro de las líneas de trabajo que pueden ubicarse bajo el amplio marco de las $\mathrm{DH}$, hay algunas facetas en las que las bibliotecas, museos y archivos pueden ser un referente: se trata de la confluencia entre web semántica y datos abiertos vinculados. La construcción de bibliotecas digitales enriquecidas con marcado semántico puede suponer una expansión muy importante en este campo.

\section{Nota}

1. El CRM (conceptual reference model) del Cidoc ofrece definiciones y una estructura formal para la descripción de los conceptos y relaciones implícitas y explícitas utilizados en la documentación del patrimonio cultural. El Cidoc (International Committee for Documentation) pertenece al ICOM (International Council for Museums).

\section{Bibliografía citada}

American Council of Learned Societies, Commission on Cyberinfrastructure for the Humanities and Social Sciences (2006). Our cultural commonwealth.

http://www.acls.org/uploadedFiles/Publications/ Programs/Our_Cultural_Commonwealth.pdf

Anderson, Sheila; Blanke, Tobias (2012). "Taking the long view: from e-Science humanities to humanities digital ecosystems". Historical social research, v. 37, n. 3, pp. 147-164. http://nbn-resolving.de/urn:nbn:de:0168-ssoar-378350

Atkins, Daniel E.; Droegemeier, Kelvin K.; Feldman, Stuart I. et al. (2003). Revolutionizing science and engineering through cyberinfrastructure: Report of the National Science Foundation. Blue-Ribbon Advisory Panel on Cyberinfrastructure, January, $84 \mathrm{pp}$.

http://www.nsf.gov/cise/sci/reports/atkins.pdf

Bia-Platas, Alejandro; Sánchez-Quero, Manuel (2001). "Diseño de un procedimiento de marcado para la automatización del procesamiento de textos digitales usando XML y TEI". En: De-la-Fuente, Pablo; Pérez, Adoración (eds.). II Jornadas bibliotecas digitales (Jbidi), Almagro, pp. 153-165. http://www.cervantesvirtual.com/nd/ark:/59851/bmcn0143

Casas-Del-Álamo, María; Redondo-Pérez, Germán; Sánchez-Bellido, Sara (eds.) (2013). Actas del Seminario intl sobre bibliotecas digitales y bases de datos especializadas para la investigación en literaturas hispánicas (Bideslite). Madrid, 4-5 julio 2011 [Papeles de trabajo de Dialogyca BDDH; 3]. http://eprints.ucm.es/21207

Estalella, Adolfo; Ardévol, Elisenda (2011). "e-research: desafíos y oportunidades para las ciencias sociales". Convergencia. Revista de ciencias sociales, v. 18, n. 55, pp. 87-111. http://www.redalyc.org/articulo.oa? id $=10515210004$

Hobohm, Hans-Christoph (2012). “Can digital libraries generate knowledge?". Historical social research, v. 37, n. 3, pp. 218-229. http://nbn-resolving.de/urn:nbn:de:0168-ssoar-378935

Kirschenbaum, Matthew (2012). "Digital humanities as/is a tactical term". In: Gold, Matthew K. (ed.). Debates in the digital humanities. Minneapolis: University of Minnesota Press, pp. 415-428.

http://dhdebates.gc.cuny.edu/debates

Kirschenbaum, Matthew (2014). "What is 'digital humanities', and why are they saying such terrible things about it?". Differences: A journal of feminist cultural studies, v. 25, n. 1. http://dx.doi.org/10.1215/10407391-2419997

Lucía-Megías, José-Manuel (2014). "La humanidades digitales: una oportunidad para los hispanistas del siglo XXI". Janus, Anexo 2, pp. 99-116.

http://www.janusdigital.es/anexos/contribucion. $h t m ? i d=49$

Maron, Nancy; Miller, Sylvia; Watkinson, Charles; Kenney, Anne (2013). "Publarians and lubishers: Role bending in the new scholarly communications ecosystem". In: Procs of the Charleston library conf.

http://dx.doi.org/10.5703/1288284315317

Oldman, Dominic; Doerr, Martin; De-Jong, Gerald; Norton, Barry; Wikman, Thomas (2014). "Realizing lessons of the last 20 years: A manifesto for data provisioning \& aggregation services for the digital humanities (a position paper)". D-lib magazine, v. 20, n. 7/8.

http://dx.doi.org/10.1045/july2014-oldman

Rodríguez-Yunta, Luis (2013). “Humanidades digitales, ¿̇una mera etiqueta o un campo por el que deben apostar las ciencias de la documentación?". Anuario ThikEPI, v. 7, pp. 37-43. http://eprints.rclis.org/19368

Rodríguez-Yunta, Luis (2014). “Indización en profundidad y aplicación de metadatos a materiales suplementarios en la edición de revistas". Anuario ThinkEPI, v. 8, pp. 207-210. http://eprints.rclis.org/23315

Rojas-Castro, Antonio (2013). “El mapa y el territorio. Una aproximación histórico-bibliográfica a la emergencia de las humanidades digitales en España". Caracteres, v. 2, n. 2. http://revistacaracteres.net/revista/vol2n2noviembre2013/ el-mapa-y-el-territorio

Romero-Frías, Esteban (2014). "Ciencias sociales y humanidades digitales: una visión introductoria”. En: Romero-Frías, Esteban; Sánchez-González, María (eds.). Ciencias sociales y humanidades digitales. Técnicas, herramientas y experiencias de e-research e investigación en colaboración. La Laguna: Sociedad Latina de Comunicación Social [CAC, Cuadernos artesanos de comunicación; 61].

http://www.cuadernosartesanos.org/\#61

Rydberg-Cox, Jeffrey A. (2006). Digital libraries and the challenges of digital humanities. Oxford: Chandos Publishing.

Schmidt, Desmond (2012). "The role of markup in the digital humanities". Historical social research, v. 37, n. 3, pp. 125-146. http://nbn-resolving.de/urn:nbn:de:0168-ssoar-378369

Spence, Paul (2014a). "Centros y fronteras: el panorama internacional". Janus, Anexo 1, pp. 37-61. http://www.janusdigital.es/anexos/contribucion. $h t m$ ?id=6

Spence, Paul (2014b). "La investigación humanística en la era digital: mundo académico y nuevos públicos". Janus, Anexo 2, pp. 117-131. 
http://www.janusdigital.es/anexos/contribucion.htm?id=50

Vandegrift, Micah; Varner, Stewart (2013). "Evolving in common: creating mutually supportive relationships between libraries and the digital humanities". Journal of $\mid i-$ brary administration, v. 53, n. 1, pp. 67-78. http://dx.doi.org/10.1080/01930826.2013.756699

Van-Zundert, Joris (2012). "If you build it, will we come? Large scale digital infrastructure as a dead end for digital humanities". Historical social research, v. 37, n. 3, pp. 165-186. http://nbn-resolving.de/urn:nbn:de:0168-ssoar-378903

\section{Recursos e instituciones citados}

Alabama Digital Humanities Center http://www.lib.ua.edu/digitalhumanities

Alliance of Digital Humanities Organizations http://adho.org

American Council of Learned Societies http://www.acls.org

Artnodes

http://journals.uoc.edu/index.php/artnodes

Asociación Argentina de Humanidades Digitales http://aahd.com.ar

Atlas de ciencias sociales y humanidades digitales http://grinugr.org/mapa

Biblioteca Saavedra Fajardo de Pensamiento Político Hispánico http://www.saavedrafajardo.org

Bidiso - Biblioteca Digital Siglo de Oro http://www.bidiso.es

Bieses - Bibliografía de escritoras españolas http://www.bieses.net

Caracteres. Estudios culturales y críticos de la esfera digital http://revistacaracteres.net

Center for Digital Scholarship - Brown University Library http://library.brown.edu/cds

CenterNet

http://digitalhumanities.org/centernet

Clarisel - Bases de datos de literatura http://clarisel.unizar.es

DHCommons

http://dhcommons.org

Dialogyca BDDH - Biblioteca Digital de Diálogo Hispánico http://www.ucm.es/info/dialogycabddh

Digital classicist

http://www.digitalclassicist.org

Digital Humanities Center - Columbia University Libraries http://library.columbia.edu/locations/dhc.html

Digital humanities quarterly http://www.digitalhumanities.org/dhq

Digital Humanities Summer Institute http://www.dhsi.org
Digital humanities wiki http://digitalhumanities.pbworks.com

Digital medievalist. The journal of the digital medievalist community http://www.digitalmedievalist.org/journal

Digital scholarship in the humanities. Exploring the digital humanities

http://digitalscholarship.wordpress.com

Digithum - Les humanitats en l'era digital http://journals.uoc.edu/index.php/digithum

DiRT - Directory digital research tools http://dirtdirectory.org

European Association for Digital Humanities http://eadh.org

Filindig - Filología e innovación en humanidades digitales http://filindig.hypotheses.org

Filología digital

http://fildigital.hypotheses.org

Geolnformatica

http://link.springer.com/journal/10707

Grinugr

http://grinugr.org

Grup de Recerca Art, Arquitectura i Societat Digital - UB http://www.artyarqdigital.com

Grupo de Gestión del Conocimiento y Nuevas Tecnologías - UCLM

http://www.uc/m.es/grupo/gcynt

Huma-Num

http://www.huma-num.fr

Humanidades digitales hispánicas

http://www.humanidadesdigitales.org

Isidore

http://www.rechercheisidore.fr

Janus. Estudios sobre el Siglo de oro

http://www.janusdigital.es

Journal of digital humanities

http://journalofdigitalhumanities.org

Laboratoire de cultures et humanités digitales - Université de Lausanne

http://unil.ch/ladhul/home.html

Laboratorio de Innovación en Humanidades Digitales UNED

http://linhd.uned.es

Literary and linguistic computing

http://Ilc.oxfordjournals.org

Máster en humanidades digitales, UAB

http://goo.gl/Gn5uai

MediaLab USAL

http://medialab.usal.es/blog/humanidades-digitales 
Office of Digital Humanities

http://www.neh.gov/divisions/odh

Open Humanities Press

http://openhumanitiespress.org

OpenEdition

http://www.openedition.org

Phebo-Poesía hispánica en el bajo barroco http://www.phebo.es

Red Aracne, Red de Humanidades Digitales y Letras Hispánicas http://www.red-aracne.es

Red de Humanidades Digitales

http://humanidadesdigitales.net
Roy Rosenzweig Center for History and New Media http://chnm.gmu.edu

Sociedad Española de Arqueología Virtual http://www.arqueologiavirtual.com

TEI Text Encoding Initiative http://www.tei-c.org/index.xml

Texas Digital Humanities Consortium http://www.txdhc.org

Zotero group - Digital Humanities Education https://www.zotero.org/groups/digital_humanities_ education

\section{$1{ }^{a}$ CONFERENCIA INTERNACIONAL SOBRE INDUSTRIA Y MERCADO DE LA INFORMACIÓN (Confimi) \\ Madrid, 5-6 de febrero de 2015}

\section{Organizada por:}

- El profesional de la información;

- Biblioteca de la Universidad Complutense de Madrid; y

- Casa del Lector, de la Fundación Germán Sánchez Ruipérez.

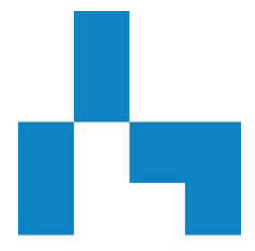

CASA DEL LECTOR

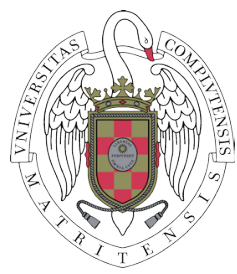

\section{El profesional de la} información

\section{Dirigida a:}

- responsables de adquisiciones y profesionales de la información de universidades, consorcios, bibliotecas virtuales, redes cooperativas, centros de investigación, empresas y administraciones;

- investigadores y profesores de biblioteconomía, documentación y ciencias de la información; y

- empresas proveedoras de contenido, tanto productoras como distribuidoras.

\section{Objetivos:}

- analizar problemáticas del mercado de la información (costes, valor, beneficios, análisis beneficio/coste, productividad, política de adquisiciones, suscripciones, evolución y tendencias...); y

- evaluar la oferta de nuevos productos y servicios que ofrecen los proveedores.

\section{Más información}

http://confimi.info

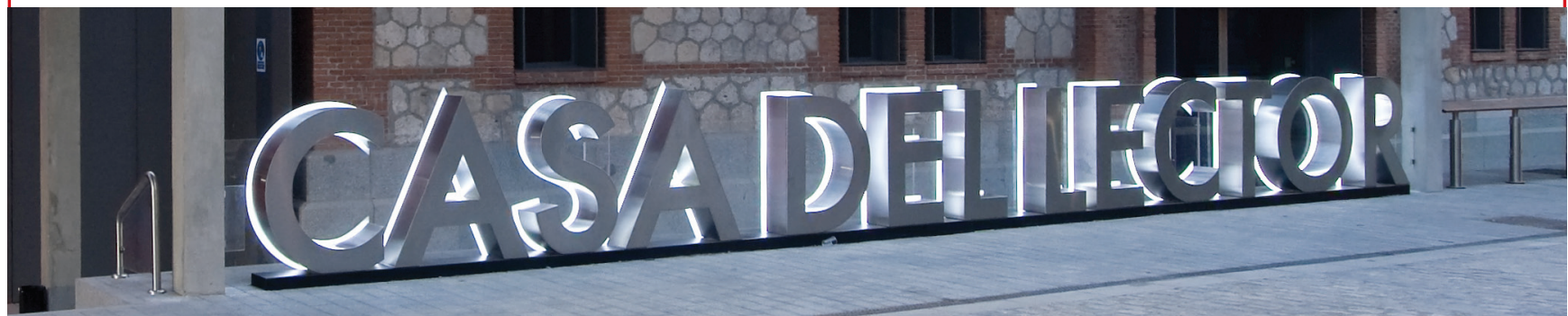

